\title{
719 XTX301, A PROTEIN-ENGINEERED IL-12, EXHIBITS TUMOR-SELECTIVE ACTIVITY IN MICE WITHOUT PERIPHERAL TOXICITIES AND IS WELL TOLERATED IN NON-HUMAN PRIMATES
}

Ekta Patel ${ }^{*}$, Natalia Malkova, Sallyann Vu, Rebekah O'Donnell, Manoussa Fanny, Wilson Guzman, Parker Johnson, Megan McLaughlin, Oleg Yerov, Kurt Jenkins, Hanumantha Rao Madala, Caitlin O'Toole, Magali Pederzoli-Ribeil, Jia Chen, Benjamin Nicholson, Bill Avery, Huawei Qiu, Ronan O'Hagan, Jennifer O'Neil. Xilio Therapeutics, Waltham, MA, United States

Background Interleukin-12 (IL-12) is a proinflammatory cytokine which bridges innate and adaptive immunity via induction of $\mathrm{T}$ helper 1 differentiation and promoting cytolytic activity of natural killer and T cells. IL-12 has demonstrated potent antitumor activity in syngeneic mouse models and promising anti-tumor efficacy in humans. However, development of IL-12 has been limited by severe systemic toxicities. To overcome toxicity and improve the therapeutic index of IL-12, we employed protein engineering to generate XTX301, a highly potent, half-life extended and masked IL-12. The masking domain of XTX301 is designed to pharmacologically inactivate IL-12 systemically and render an active IL-12 moiety upon cleavage by proteases that are enriched in the tumor microenvironment.

Methods We conducted experiments to assess the binding, bioactivity, safety, and anti-tumor efficacy of XTX301. Binding interactions were measured via SPR, bioactivity was measured using STAT-4 phosphorylation in a reporter cell line, and IFN-g production was assessed in human PBMCs via ELISA. Anti-tumor efficacy and pharmacodynamics were assessed in MC38 and B16F10 syngeneic tumor mouse models using a XTX301 murine surrogate, mXTX301. Safety and pharmacokinetics of XTX301 were evaluated in non-human primates (NHP).

Results XTX301 showed no detectable binding to the high affinity IL12RB2 demonstrating that the masking domain indeed prevents interaction with the receptor. Upon cleavage of the masking domain by relevant proteases, binding was observed and was comparable to XTX300 unmasked control. Likewise, restoration of activity upon proteolytic cleavage was observed in an IL-12-dependent reporter gene assay and in primary human PBMCs. Human IL-12 does not cross react with mouse IL-12 receptors; hence a murine surrogate (mXTX301) was created for in vivo anti-tumor efficacy evaluation. A single dose of mXTX301 demonstrated up to $90 \%$ tumor growth inhibition in an inflamed MC38 and noninflamed B16F10 syngeneic mouse models. mXTX301 induced a $\sim 3$ fold increase in IFN-g in tumors compared to vehicle control and $\sim 150$ fold less peripheral IFN-g compared to mXTX300. XTX301 exhibits minimal elevation in liver enzymes and a 50-fold improvement in tolerability compared to XTX300, in a repeat dose NHP safety study.

Conclusions Our data demonstrates that both XTX301 and mXTX301 are inactive when in masked form and become activated upon proteolytic cleavage to exert bioactivity comparable to recombinant IL-12. For efficacy, mXTX301 demonstrated tumor selective activity in syngeneic mouse models. XTX301 was well tolerated in repeat dose NHP safety study. In conclusion, XTX301 has potential for exerting potent antitumor activity with a favorable tolerability profile. 